\title{
A boundary element method for wave scattering in fluid-saturated porous rocks
}

\author{
A. Furukawa ${ }^{1}$, T. Saitoh ${ }^{2} \&$ S. Hirose ${ }^{1}$ \\ ${ }^{1}$ Department of Mechanical and Environmental Informatics, \\ Tokyo Institute of Technology, Japan \\ ${ }^{2}$ Division of Environmental Engineering Science, \\ Gunma University, Japan
}

\begin{abstract}
Wave analysis in rocks is widely used in earthquake engineering and geophysical exploration. Rocks under the ground include pores and cracks which are saturated with pore fluid. Waves which propagate in the rocks are affected by these cracks and pore fluid. Therefore, in the numerical simulation of the rocks, it is necessary to consider the effects of both anisotropy and pore fluid. Biot has been proposed as a mechanical model for describing the behavior of such a rock, and this model forms a foundation for wave analysis of general anisotropic fluid-saturated porous solids. This study aims to develop a boundary element method for wave scattering in general anisotropic fluid-saturated porous solids. Formulation is based on the following two kinds of boundary integral equations: one is those for displacement of the solid skeleton and the other is for fluid pressure. Green's function for wave analysis in general anisotropic fluid-saturated porous solids is derived by using Radon and Fourier transforms in space. Some numerical examples show the validity of our proposed method.

Keywords: boundary element method, fluid-saturated porous solid, anisotropy.
\end{abstract}

\section{Introduction}

Wave propagation and scattering in rocks have been discussed in earthquake engineering and exploration geophysics. Rocks in underground have the following important properties: anisotropy, dispersion, and attenuation. Anisotropy is generated by crystal preferred orientation of the rocks and aligned microcracks in the rocks. Dispersion and attenuation are generated by porous structure of the rocks 
and existence of pore fluid. Therefore, it is desirable that the rocks be considered as a general anisotropic fluid-saturated porous solid.

A wave theory of fluid-saturated porous solids has been proposed by Biot $[1,2]$, and the theory considering anisotropic effects was subsequently presented [3]. The mechanical model proposed by Biot is called Biot's model, and there are various expanded models which have been derived from Biot's original one. Recently, properties of waves propagating in anisotropic fluid-saturated porous solids are discussed by many researchers [4-6].

In recent years, a novel time-domain BEM, convolution quadrature boundary element method (CQ-BEM), has been proposed [7]. The CQ-BEM is a timedomain BEM whose boundary integral equations (BIEs) are discretized by a convolution quadrature method (CQM). The CQM evaluates Riemann convolution $f(t) * g(t)$ numerically, and requires Laplace transform of the function $f(t)$. Therefore, in CQ-BEM formulation, time-domain BIEs are solved using Laplacedomain fundamental solutions. Main advantages of CQ-BEM are to produce stable numerical solutions with small time increments compared with the conventional one, and to deal with waves affected by dispersion and dissipation in time-domain.

Boundary element methods for poroelastodynamics based on Biot's model have been developed in the last few decades. Domigues proposed a frequencydomain BEM formulation which describes dynamic behavior by using two kinds of boundary values, i.e. solid displacement and fluid pressure [8]. On the other hand, time-domain BEMs for Biot's model have been developed with the advent of the CQ-BEM $[9,10]$. These formulations solved time-domain BIEs with respect to solid displacement and fluid pressure as boundary values. However, these previous BEM formulations can be applied to isotropic fluid-saturated porous solids only, and the research on BEM for elastodynamics in general anisotropic fluid-saturated porous solids has not been carried out, as long as the authors know.

In this paper, a CQ-BEM for two-dimensional wave scattering in general anisotropic fluid-saturated porous solids is presented, and the validity of our proposed method is confirmed. In the following sections, firstly, the theory of Biot's model is summarized, and CQ-BEM formulation is subsequently illustrated. Numerical examples are presented after these descriptions. Throughout this paper, summation convention is valid for repeated indices.

\section{Biot's theory}

This section introduces Biot's model [3], that is well known as a mechanical model for general anisotropic fluid-saturated porous solids. The required assumptions of the Biot's theory are as follows: (1) Fluid-saturated porous solid consists of solid skeleton and pore fluid. (2) Infinitesimal transformations occur between the reference and current states of deformation. (3) The wavelength is large compared with the dimensions of macroscopic values. (4) The conditions are isothermal. (5) The fluid is viscous. (6) The fluid flows through the porous skeleton according to Darcy's law. Note that the presented model focuses on the anisotropy due to a preferential alignment of the pores or cracks. This formulation can treat anisotropic 
effects of not only elasticity of the solid skeleton but also permeability of pore fluid. In this section, the subscript written by a small letter takes the values from 1 to 3 .

Constitutive equations for the Biot's model are given by the following equations:

$$
\begin{aligned}
& \sigma_{i j}=A_{i j k l} u_{k, l}+\alpha_{i j} M w_{k, k}, \\
& p=-\alpha_{k l} M u_{k, l}-M w_{k, k}
\end{aligned}
$$

where $\sigma_{i j}$ is total stress of the solid, and $p$ is fluid pressure. $u_{i}$ and $w_{i}$ represent displacement of the solid skeleton and flow of the fluid relative to the solid in the unit section, respectively. In addition, ()$_{, i}=\partial / \partial x_{i}$. M is Biot's elastic modulus, and $\alpha_{i j}$ is Biot's effective-stress coefficients for general anisotropy. Moreover, $A_{i j k l}$ denotes elastic tensor of undrained porous solid expressed as follows:

$$
A_{i j k l}=C_{i j k l}+\alpha_{i j} \alpha_{k l} M
$$

where $C_{i j k l}$ is elastic tensor of drained porous solid, i.e. solid skeleton.

Equations of motion for the Biot's model are expressed as follows:

$$
\begin{aligned}
& \sigma_{i j, j}+\rho b_{i}=\rho \ddot{u}_{i}+\rho_{f} \ddot{w}_{i}, \\
& p_{, i}+\rho_{f} c_{i}=-\rho_{f} \ddot{u}_{i}-m_{i j} \ddot{w}_{j}-\eta r_{i j} \dot{w}_{j}
\end{aligned}
$$

where $\rho_{f}$ and $\rho$ are density of pore fluid and the porous solid. The density of the porous solid $\rho$ is given by the following equation: $\rho=(1-\beta) \rho_{s}+\beta \rho_{f}$ where $\beta$ and $\rho_{s}$ are porosity and density of the solid skeleton, respectively. $b_{i}$ and $c_{i}$ are body force of the porous solid and pore fluid, respectively, and $\left(\dot{)}=\partial / \partial t . m_{i j}\right.$ is mass matrix determined by geometry of pores. $\eta$ is fluid viscosity, and $r_{i j}$ denotes the flow resistivity matrix which is same with the inverse matrix of the permeability matrix based on the Darcy's law.

Characteristics of wave propagation in general anisotropic fluid-saturated porous solids are as follows: The solids generate four body waves; i.e., quasi-fast compressional wave (qP1), quasi-slow compressional wave (qP2), and quasi-shear waves (qS1 and qS2). In addition, phase velocities of the waves depend on not only propagation direction but also frequency, and the viscosity $\eta$ effects great changes on the behavior of $\mathrm{qP} 2$ wave.

\section{Boundary element method}

In this section, a formulation of convolution quadrature boundary element method (CQ-BEM) for two-dimensional wave scattering in the anisotropic Biot's model is presented. The contents are as follows: Firstly, the BEM formulation based on time-domain boundary integral equations (BIEs) is described. Secondly, time- and spatial discretizations of the BIEs are conducted. Finally, fundamental solutions for the target problems are derived. In this section, without notice, the subscripts written by small and capital letters range 1 to 2 and 1 to 3 , respectively. 


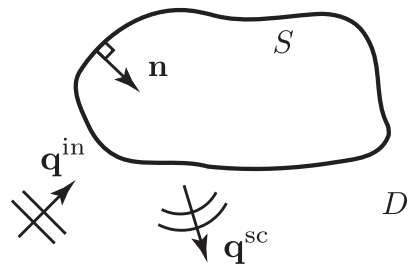

Figure 1: Wave scattering in general anisotropic fluid-saturated porous media.

\subsection{Time-domain boundary integral equations}

In our proposed method, the following boundary value vectors, generalized displacement $q_{I}$ and traction $s_{I}$, are used:

$$
\begin{aligned}
& q_{I}=\left\{\left\{u_{i}\right\}^{\mathrm{T}}, p\right\}^{\mathrm{T}}, \\
& s_{I}=\left\{\left\{t_{i}\right\}^{\mathrm{T}}, p_{n}\right\}^{\mathrm{T}}=\left\{\left\{\sigma_{i j} n_{j}\right\}^{\mathrm{T}}, w_{j} n_{j}\right\}^{\mathrm{T}}
\end{aligned}
$$

where $t_{i}$ is the traction component of the solid, and $p_{n}$ is normal derivative of the pressure given by $p_{n}=\partial p / \partial n$. In addition, $n_{i}$ is the unit normal vector on the boundary, and \{\}$^{\mathrm{T}}$ denotes transpose of a vector. Our target is wave scattering as shown in Fig. 1, and its initial conditions at point $\mathbf{x}$ are given as follows:

$$
q_{I}(\mathbf{x}, t)=0, \dot{q}_{I}(\mathbf{x}, t)=0, t=0 .
$$

Considering wave scattering in infinite domain $D$ whose boundary is $S$, a timedomain BIEs for Biot's model are expressed as follows:

$$
\begin{aligned}
C(\mathbf{x}) q_{I}(\mathbf{x}, t)= & q_{I}^{\mathrm{in}}(\mathbf{x}, t)+\int_{S} U_{I K}(\mathbf{x}, \mathbf{y}, t) * s_{K}(\mathbf{y}, t) d S(\mathbf{y}) \\
& -\int_{S} W_{I K}(\mathbf{x}, \mathbf{y}, t) * q_{K}(\mathbf{y}, t) d S(\mathbf{y})
\end{aligned}
$$

where

$$
C(\mathbf{x})=\left\{\begin{array}{cll}
1 & : & \mathbf{x} \in D \\
1 / 2 & : & \mathbf{x} \in S \\
0 & : & \text { otherwise }
\end{array}\right.
$$

In Eq. (9), $*$ denotes the Riemann convolution, and $q_{I}^{\text {in }}(\mathbf{x}, t)$ represents generalized displacement for the incident wave. Moreover, $U_{I K}(\mathbf{x}, \mathbf{y}, t)$ and $W_{I K}(\mathbf{x}, \mathbf{y}, t)$ represent time-domain fundamental solutions and its double layer kernels, respectively. 


\subsection{Discretization of boundary integral equations}

Time- and spatial discretization of the BIEs shown in Eq. (9) gives an algebraic equation which can be solved numerically. The presented discretizations are applied by the following manners: Convolution quadrature method (CQM) based on the backward difference formula is used for time-discretization. Collocation method with constant shape function is used for spatial discretization.

Approximation formula of the CQM is given by the following equation:

$$
f(n \Delta t) * g(n \Delta t) \simeq \sum_{k=0}^{n} \omega_{n-k}(\Delta t) g(k \Delta t)
$$

where $\Delta t$ represents time increment, and $\omega_{m}$ is the weight function described as follows:

$$
\omega_{m}(\Delta t) \simeq \frac{R^{-m}}{L} \sum_{l=0}^{L-1} \hat{f}\left(\frac{\gamma\left(z_{l}\right)}{\Delta t}\right) \mathrm{e}^{-2 \pi \mathrm{i} m \frac{l}{L}} .
$$

The CQM parameters with an error magnitude $O(\epsilon)$ are written as follows:

$$
\gamma(z)=\sum_{i=1}^{k} \frac{1}{i}(1-z)^{i}, z_{l}=R \mathrm{e}^{-2 \pi \mathrm{i} \frac{l}{L}}, R=\epsilon^{\frac{1}{2 L}} .
$$

Note that Eq. (13) corresponds to the backward differentiation formulas of order $k$. The form of the weight function shown in Eq. (12) is same with that of discretized Fourier transform (DFT). In numerical computation, fast Fourier transform (FFT) can be applied to this calculation if $L$ is equal to the number of total time step $N_{t}$.

Taking the limit process $\mathbf{x} \in D \rightarrow \mathbf{x} \in S$ and conducting the above-mentioned discretizations, algebraic equation for wave scattering analysis is obtained:

$$
\begin{gathered}
\sum_{N=1}^{N_{e}}\left[\left\{\frac{1}{2} \delta_{M N} \delta_{I K}+B_{M N ; I K}^{(0)}\right\} q_{N ; K}^{(n)}-A_{M N ; I K}^{(0)} s_{N ; K}^{(n)}\right] \\
=q_{M ; I}^{\mathrm{in}(n)}+\sum_{k=1}^{n-1} \sum_{N=1}^{N_{e}}\left[A_{M N ; I K}^{(n-k)} s_{N ; K}^{(k)}-B_{M N ; I K}^{(n-k)} q_{N ; K}^{(k)}\right] \\
M=1,2, \cdots, N_{e}, n=1,2, \cdots, N_{t}
\end{gathered}
$$

where $\delta_{i k}$ is Kronecker delta, and $N_{e}$ represents the number of boundary elements. Moreover, $A_{M N ; I K}^{(k)}$ and $B_{M N ; I K}^{(k)}$ are influence functions expressed as follows:

$$
\begin{aligned}
& A_{M N ; I K}^{(m)}=\frac{R^{-m}}{L} \sum_{l=0}^{L-1}\left[\int_{S^{N}} \hat{U}_{I K}\left(\mathbf{x}^{M}, \mathbf{y}, s_{l}\right) d S(\mathbf{y})\right] \mathrm{e}^{-2 \pi \mathrm{i} m \frac{l}{L}}, \\
& B_{M N ; I K}^{(m)}=\frac{R^{-m}}{L} \sum_{l=0}^{L-1}\left[\int_{S^{N}} \hat{W}_{I K}\left(\mathbf{x}^{M}, \mathbf{y}, s_{l}\right) d S(\mathbf{y})\right] \mathrm{e}^{-2 \pi \mathrm{i} m \frac{l}{L}}
\end{aligned}
$$


where $s_{l}=\gamma\left(z_{l}\right) / \Delta t$, and $\hat{U}_{I K}(\mathbf{x}, \mathbf{y}, s)$ and $\hat{W}_{I K}(\mathbf{x}, \mathbf{y}, s)$ are the Laplace-domain fundamental solutions and its double layer kernels, respectively. Substituting prescribed boundary conditions into Eq. (14) and solving the resulting algebraic equation at each time step, the unknown boundary values can be obtained.

\subsection{Fundamental solutions for anisotropic Biot's model}

Fundamental solutions for our proposed formulation are presented in this section. Derivation procedure of the fundamental solutions presented here is almost same with that of fundamental solutions for elastodynamics in general anisotropic solids [11]. Note that our proposed method requires Laplace-domain solutions because of implementation of the CQM.

Laplace-domain fundamental solutions $\hat{U}_{I K}(\mathbf{x}, \mathbf{y}, s)$ are given by the following equation:

$$
\hat{L}_{P Q} \hat{U}_{Q K}(\mathbf{x}, \mathbf{y}, s)=-\delta(\mathbf{x}-\mathbf{y}) \delta_{P K}
$$

where $\delta(\cdot)$ is Dirac delta function, and $s$ is Laplace parameter. The components of matrix $\hat{L}_{I K}$ are expressed as follows:

$$
\hat{L}_{I K}=\left[\begin{array}{cc}
{\left[C_{i \beta k \gamma} \partial_{\beta} \partial_{\gamma}-\tilde{\rho}_{i k} s^{2}\right]} & \left\{-\tilde{\alpha}_{i \beta} \partial_{\beta}\right\} \\
\left\{\tilde{\alpha}_{k \gamma} \partial_{\gamma}\right\}^{T} & -\frac{1}{s^{2}} Y_{\beta \gamma}^{-1} \partial_{\beta} \partial_{\gamma}+\frac{1}{M}
\end{array}\right]
$$

where $\partial_{\beta}=\partial / \partial x_{\beta}$, and

$$
\tilde{\alpha}_{i \beta}=\alpha_{i \beta}-\rho_{f} Y_{i \beta}^{-1}, \tilde{\rho}_{i k}=\rho \delta_{i k}-\rho_{f}^{2} Y_{i k}^{-1}, Y_{i k}=m_{i k}+\frac{1}{s} \eta r_{i k} .
$$

It should be emphasized that the subscripts written by small and capital letters range 1 to 3 and 1 to 4, respectively in Eqs (17)-(19). Moreover, the subscript written by a Greek character takes the values from 1 to 2 in the above-mentioned equations. Solving Eq. (17) using Radon and Fourier transforms in space, the following fundamental solutions are obtained:

$$
\begin{aligned}
& \hat{U}_{i k}(\mathbf{x}, \mathbf{y}, s)=\frac{1}{4 \pi^{2}} \int_{|\mathbf{p}|=1} \sum_{\alpha=1}^{4} \Lambda_{i k}^{\alpha}(\mathbf{r}, s, \mathbf{p}) \Phi_{\alpha}(\mathbf{r}, s, \mathbf{p}) d L(\mathbf{p}), \\
& \hat{U}_{i 3}(\mathbf{x}, \mathbf{y}, s)=\frac{1}{4 \pi^{2}} \int_{|\mathbf{p}|=1} \sum_{\alpha=1}^{4} \Lambda_{i 4}^{\alpha}(\mathbf{r}, s, \mathbf{p}) \Psi_{\alpha}(\mathbf{r}, s, \mathbf{p}) d L(\mathbf{p}), \\
& \hat{U}_{3 k}(\mathbf{x}, \mathbf{y}, s)=\frac{1}{4 \pi^{2}} \int_{|\mathbf{p}|=1} \sum_{\alpha=1}^{4} \Lambda_{4 k}^{\alpha}(\mathbf{r}, s, \mathbf{p}) \Psi_{\alpha}(\mathbf{r}, s, \mathbf{p}) d L(\mathbf{p}), \\
& \hat{U}_{33}(\mathbf{x}, \mathbf{y}, s)=\frac{1}{4 \pi^{2}} \int_{|\mathbf{p}|=1} \sum_{\alpha=1}^{4} \Lambda_{44}^{\alpha}(\mathbf{r}, s, \mathbf{p}) \Phi_{\alpha}(\mathbf{r}, s, \mathbf{p}) d L(\mathbf{p})
\end{aligned}
$$


where $\mathbf{r}=\mathbf{x}-\mathbf{y}$, and

$$
\begin{gathered}
\Lambda_{I K}^{\alpha}(\mathbf{r}, s, \mathbf{p})=k_{\alpha} \frac{\left(\left.\operatorname{adj}[\tilde{\hat{\mathbf{L}}}]\right|_{k=k_{\alpha}}\right)_{I K}}{\left.\partial^{[k]} \operatorname{det}[\underline{\hat{\mathbf{L}}}]\right|_{k=k_{\alpha}}}, I, K=1,2,3,4 \\
\Phi_{\alpha}(\mathbf{r}, s, \mathbf{p})=\mathrm{e}^{-\mathrm{i} k_{\alpha}|\mathbf{p} \cdot \mathbf{r}|} E_{1}\left(-\mathrm{i} k_{\alpha}|\mathbf{p} \cdot \mathbf{r}|\right)+\mathrm{e}^{\mathrm{i} k_{\alpha}|\mathbf{p} \cdot \mathbf{r}|}\left\{E_{1}\left(\mathrm{i} k_{\alpha}|\mathbf{p} \cdot \mathbf{r}|\right)+\mathrm{i} \pi\right\} \\
\Psi_{\alpha}(\mathbf{r}, s, \mathbf{p})=\operatorname{sgn}(\mathbf{p} \cdot \mathbf{r})\left[-\mathrm{e}^{-\mathrm{i} k_{\alpha}|\mathbf{p} \cdot \mathbf{r}|} E_{1}\left(-\mathrm{i} k_{\alpha}|\mathbf{p} \cdot \mathbf{r}|\right)\right. \\
\left.+\mathrm{e}^{\mathrm{i} k_{\alpha}|\mathbf{p} \cdot \mathbf{r}|}\left\{E_{1}\left(\mathrm{i} k_{\alpha}|\mathbf{p} \cdot \mathbf{r}|\right)+\mathrm{i} \pi\right\}\right]
\end{gathered}
$$

In Eq. (24), $(\breve{)}$ and $(\check{)}$ denote spatial Radon and Fourier transforms. Moreover, $E_{1}(\cdot)$ in Eqs (25) and (26) is the exponential integral, and $k_{\alpha}$ is the wavenumber of the four body waves obtained by solving the following eigen equation:

$$
\operatorname{det}[\check{\hat{\mathbf{L}}}]=C_{1} k_{\alpha}^{8}+C_{2} k_{\alpha}^{6}+C_{3} k_{\alpha}^{4}+C_{4} k_{\alpha}^{2}+C_{5}=0, \operatorname{Im}\left[k_{\alpha}\right]>0
$$

where $C_{1}, C_{2}, \cdots, C_{5}$ are coefficients.

In Eqs (20)-(23), the integrals over the unit circle $(|\mathbf{p}|=1)$ are implemented as a consideration of the interference of the body waves propagating in every direction. Moreover, the summation with respect to $\alpha$ denotes the superposition of the four body waves. In this study, integrals over the unit circle are evaluated numerically using double-exponential transformation [12]. Note that the computational cost is quite high because the number of sampling points of these integrals is set to be 1,400 .

The double layer kernels $\hat{W}_{I K}(\mathbf{x}, \mathbf{y}, s)$ are given by the following equations:

$$
\hat{W}_{I K}(\mathbf{x}, \mathbf{y}, s)=\hat{B}_{K J}^{\mathbf{y}} \hat{U}_{I J}(\mathbf{x}, \mathbf{y}, s)
$$

where $\hat{B}_{K J}^{\mathbf{y}}$ is generalized traction operator shown as follows:

$$
\hat{B}_{I K}^{\mathbf{y}}=\left[\begin{array}{cc}
{\left[-C_{i j k l} n_{j}(\mathbf{y}) \partial_{l}\right]} & \left\{-\alpha_{i j} n_{j}(\mathbf{y})\right\} \\
\left\{-\rho_{f} Y_{k l}^{-1} n_{l}(\mathbf{y})\right\}^{\mathrm{T}} & \frac{1}{s^{2}} Y_{j l}^{-1} n_{j}(\mathbf{y}) \partial_{l}
\end{array}\right] .
$$

In Eqs (29), $n_{i}(\mathbf{y})$ represents the unit normal vector at the point $\mathbf{y}$ on the boundary $S$.

\section{Numerical examples}

In this section, wave scattering by a cavity is solved using the proposed CQ-BEM. Analytical model illustrated in Fig. 2 consists of a cavity whose radius is $a$, and a source point $\mathbf{y}^{\mathrm{src}}$ is put at $\{-0.25 a,-0.25 a\}^{\mathrm{T}}$. Observation points are located on the line, i.e. $x_{1}=-2.6 a$ and $0<x_{3}<2.5 a$. The material of the domain 


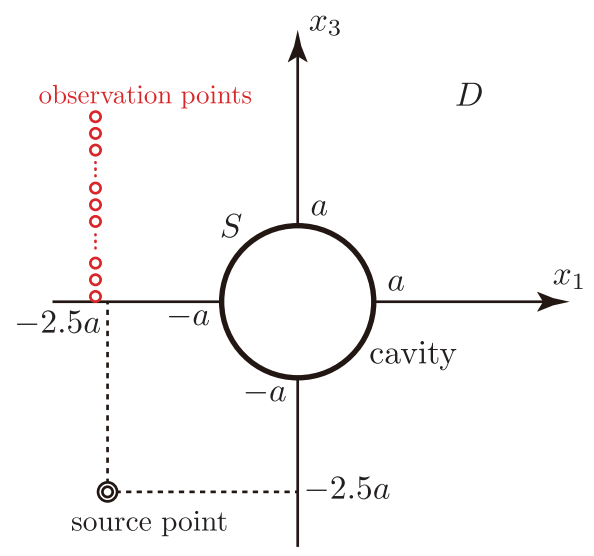

Figure 2: Numerical model for wave scattering analysis.
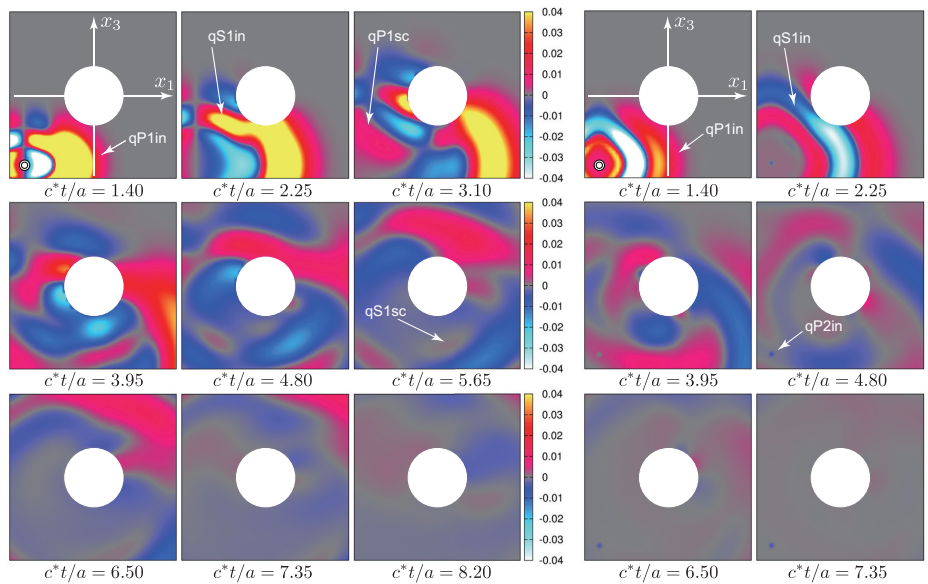

(a) displacement component $u_{1}$.

(b) fluid pressure $p$.

Figure 3: Time histories of the total wave field around a cavity $(\eta \neq 0)$.

$D$ is assumed to be brine saturated sandstone which is known as a transversely isotropic medium [4]. The number of boundary elements and total time step are set to $N_{e}=32$ and $N_{t}=512$, respectively. Time increment $\Delta t$ is given by $c^{*} \Delta t / a=0.05$ where $c^{*}=\sqrt{C_{66} / \rho_{s}}$ and $C_{66}$ is a component of elastic tensor in Voigt notation. In the presented analysis, incident wave is expressed by the following equations:

$$
\begin{aligned}
& q_{I}^{\mathrm{in}}(\mathbf{x}, t)=U_{I K}\left(\mathbf{x}, \mathbf{y}^{\mathrm{src}}, t\right) * p_{K}^{\mathrm{src}}(t), \\
& p_{K}^{\mathrm{src}}(t)=\frac{\delta_{1 K}}{2}\left\{1-\cos \left(\frac{2 \pi t}{T}\right)\right\}\{H(t)-H(t-T)\}
\end{aligned}
$$




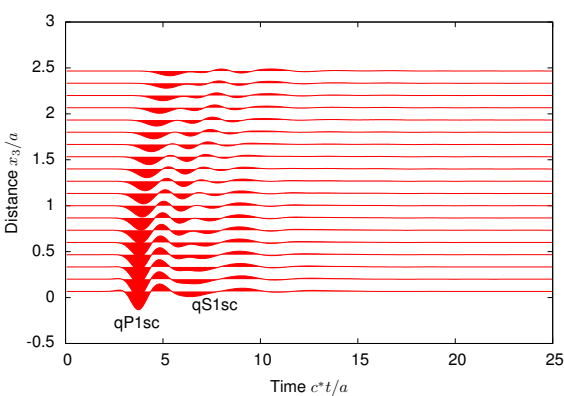

(a) displacement component $u_{1}^{\mathrm{sc}}$

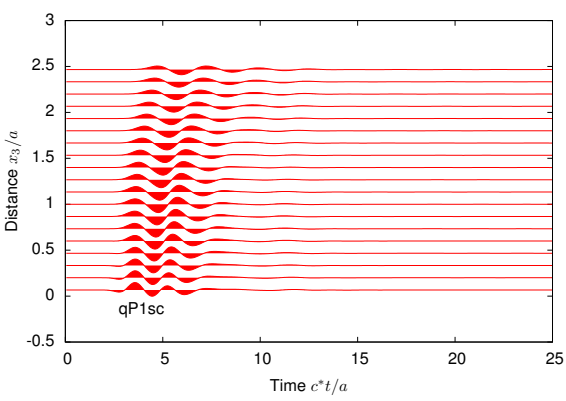

(b) fluid pressure $p^{\mathrm{sc}}$

Figure 4: Seismogram of the scattered waves on $x_{1}=2.6 a(\eta \neq 0)$.
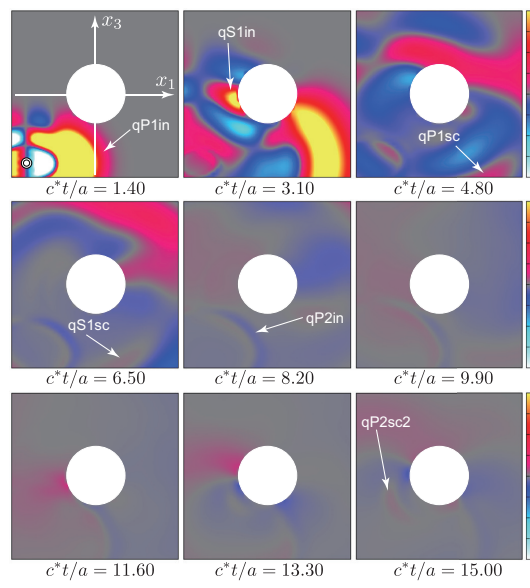

(a) displacement component $u_{1}$

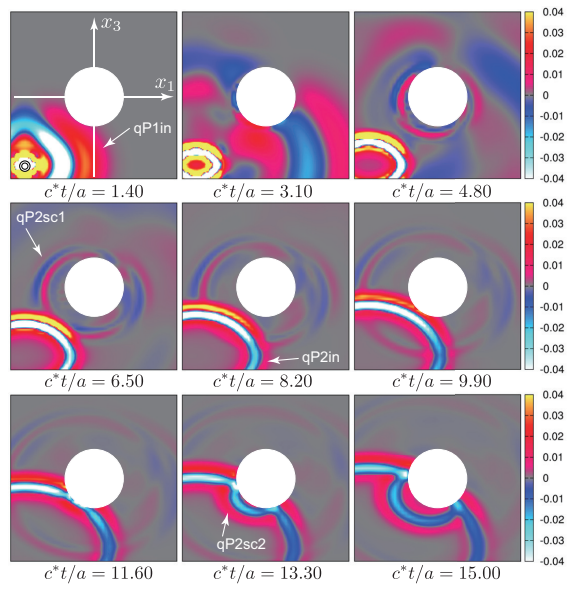

(b) fluid pressure $p$

Figure 5: Time histories of the total wave field around a cavity $(\eta=0)$.

where $H(\cdot)$ represents Heaviside function. The period of source function is set to $T=16 \Delta t$. Convolution integral shown in Eq. (30) is also computed using CQM, and its parameters described in Eqs (13) are given as follows: $\epsilon=1.0 \times 10^{-12}$ and $k=1$.

Scattered waves around a cavity are shown in Fig. 3.

In these figures, incident waves generated at the source point are propagating with complex wave surfaces because of anisotropy. When the incident waves arrived at the surface of the cavity, scattered waves, qP1sc and qS1sc waves, are generated. The interesting point is that incident $\mathrm{qP} 2$ (qP2in) wave presents a diffusive behavior because of fluid viscosity. This behavior is in good agreement with a previous research [4]. Figure 4 shows seismogram at the observation points shown in Fig. 2. 
These results confirm that waveform and arrival time of scattered waves are slightly changed.

Subsequently, further consideration of the effect of the fluid viscosity is demonstrated. Numerical results in the case that the viscosity is set to $\eta=0$ are shown in Fig. 5.

The behavior of $\mathrm{qP} 2$ wave is quite different with that of the previous results. In this case, $\mathrm{qP} 2$ wave can be observed as a wave. Therefore, fluid viscosity is an important factor for determining propagation of $\mathrm{qP} 2$ wave.

\section{Conclusions}

In this paper, a convolution quadrature boundary element method for general anisotropic fluid-saturated porous solids is presented. The proposed formulation uses CQM and collocation method for time and spatial discretizations, respectively. Wave scattering problems by a cavity in brine saturated sandstone are solved. Numerical results show the validity of our proposed method, and some important characteristics of the waves in Biot's model are confirmed.

\section{References}

[1] Biot, M.A., Theory of propagation of elastic waves in a fluid-saturated porous solid. I. Low-frequency range. The Journal of Acoustical Society of America, 28(2), pp. 168-178, 1956.

[2] Biot, M.A., Theory of propagation of elastic waves in a fluid-saturated porous solid. II. Higher frequency range. The Journal of Acoustical Society of America, 28(2), pp. 179-191, 1956.

[3] Biot, M.A., Mechanics of deformation and acoustic propagation in porous media. Journal of Applied Physics, 33(4), pp. 1482-1498, 1962.

[4] Carcione, J.M., Wave propagation in anisotropic saturated porous media: Plane-wave theory and numerical simulation. The Journal of Acoustical Society of America, 99(5), pp. 2655-2666, 1996.

[5] Sharma, M.D., Wave propagation in a general anisotropic poroelastic medium with anisotropic permeability: phase velocity and attenuation. International Journal of Solids and Structures, 41, pp. 4587-4597, 2004.

[6] Sharma, M.D., Three-dimensional wave propagation in a general anisotropic poroelastic medium: phase velocity, group velocity and polarization. Geophysical Journal International, 156, pp. 329-344, 2004.

[7] Schanz, M. \& Antes, H., Application of 'Operational quadrature methods' in time domain boundary element method. Meccanica, 32, pp. 179-186, 1997.

[8] Domínguez, J., Boundary element approach for dynamic poroelastic problems. International Journal for Numerical Methods in Engineering, 35, pp. 307-324, 1992. 
[9] Schanz, M., Application of 3D time domain boundary element formulation to wave propagation in poroelastic solids. Engineering Analysis with Boundary Elements, 25, pp. 363-376, 2001.

[10] Saitoh, T., Chikazawa, F. \& Hirose, S., Convolution quadrature time-domain boundary element method for 2-D fluid-saturated porous Media. Applied Mathematical Modelling, 38, pp. 3724-3740, 2014.

[11] Wang, C.-Y. \& Achenbach, J.D., Elastodynamic fundamental solutions for anisotropic solids. Geophysical Journal International, 118, pp. 384-392, 1994.

[12] Mori, M. \& Sugihara, M., The double-exponential transformation in numerical analysis. Journal of Computational and Applied Mathematics, 127, pp. 287-296, 2001. 